\title{
LETTERATURA E CINEMA: L'UTILIZZO DEL FILM NELLA DIDATTICA DELLA LETTERATURA ITALIANA
}

\author{
Mojca Cerkvenik, Università del Litorale, Capodistria (Slovenia), \\ mojca.cerkvenik@fhs.upr.si
}

Il cinema rappresenta una delle forme narrative più coinvolgenti, soprattutto per lo studente formatosi in costante contatto con una cultura prevalentemente visiva. Sfruttare l'esperienza e le capacità dello studente relative alla fruizione di messaggi audiovisivi per fare leva sulla motivazione è un passaggio decisivo nella predisposizione e nell'applicazione di percorsi didattici nell'ambito dell'educazione letteraria.

Il presente articolo si propone pertanto di illustrare la rilevanza, i motivi e l'utilità dell'utilizzo del cinema nell'insegnamento della letteratura, proponendo percorsi e metodi alternativi rispetto alla didattica tradizionale, che prevedono l'accostamento di testi letterari e filmici afferenti al panorama culturale italiano ed esplorano le rappresentazioni e gli immaginari che ne scaturiscono, incoraggiando l'avvicinamento a testi e mezzi espressivi in forme diverse, sia come oggetto di studio sia come opere di piacevole lettura e visione.

Le proposte didattiche formulate sono incentrate sulla scoperta congiunta dei tratti formali ed estetici di opere letterarie e cinematografiche con l'intento di individuare ed evidenziare elementi comuni, variazioni e scambi. A una visione passiva del testo filmico si sostituisce una fruizione attiva ed è in questa prospettiva che si delinea la finalità primaria dello studio di testi letterari e cinematografici: accrescere la consapevolezza critica e la capacità di decodificazione linguistica nonché il livello del gusto estetico, affinché lo studente, lettore ma anche spettatore, sia capace di scelte libere e autonome nell'universo dei messaggi audiovisivi in cui si trova immerso nella società contemporanea.

Parole chiave: letteratura italiana, cinema italiano, adattamento cinematografico, educazione letteraria, didattica della letteratura 


\section{Introduzione}

Un percorso di educazione alla letteratura efficace e stimolante, oltre a proporre contenuti curricolari tradizionali, dovrebbe essere aperto all'utilizzo di testi che gli studenti più frequentemente incontrano nell'ambito delle loro esperienze culturali extrascolastiche e verso i quali hanno sviluppato una confidenza tale da poter rispondere e partecipare con la propria immaginazione al processo di apprendimento.

Considerando l'attuale prevalere di una cultura legata all'audiovisivo più che alla parola scritta, l'uso di testi audiovisivi, in particolare dei film, nell'ambito della didattica letteraria, può offrire numerose possibilità per instaurare legami con i testi letterari generalmente proposti e può funzionare da catalizzatore per l'accrescimento della motivazione dei soggetti coinvolti nel processo di apprendimento. In aggiunta alla scoperta del piacere della lettura delle immagini, l'utilizzo del cinema può essere pensato anche come opportunità per l'educazione al senso critico e per lo sviluppo della molteplicità dei punti di vista.

Partendo da tali presupposti, nel presente contributo ci si propone di illustrare alcuni aspetti che permettono di considerare il cinema un'opportunità didattica da sfruttare nell'ambito dell'educazione letteraria e di mettere in luce quei punti di contatto tra il cinema e la letteratura che favoriscono la formulazione di percorsi didattici da utilizzare in classe.

Nella prima parte vengono presentate alcune considerazioni dei principali studiosi di educazione e didattica letteraria nel contesto italiano quali Armellini, Balboni e Freddi, che riflettono sulla possibilità di utilizzo di opere filmiche nel contesto dell'educazione letteraria, per poi esaminare quali sono le possibili ricadute operative nella realizzazione di percorsi di insegnamento e studio congiunti, in cui il cinema viene accostato alla letteratura. All'esposizione degli elementi comuni delle due forme espressive, che si inserisce nel quadro degli studi narratologici, seguono, nell'ultima parte del contributo, alcune proposte didattiche sviluppate e sottoposte a studenti universitari di italianistica presso l'Università del Litorale (Capodistria, Slovenia). Queste proposte nascono con l'obiettivo di avvicinare gli studenti alla narrativa letteraria e motivarli alla lettura favorendo lo scambio di idee per dimostrare che, nonostante l'evidente predominanza dei media digitali e dell'audiovisivo, anche l'esperienza letteraria nella sua forma più tradizionale può fornire soluzioni altrettanto interessanti e importanti rispetto alle loro necessità e aspettative. 


\section{Il cinema come opportunità didattica nel contesto dell'educazione letteraria}

L'educazione alla letteratura dovrebbe configurarsi come un progetto condiviso tra studente e insegnante che vede come obiettivi sia la scoperta del piacere di leggere che l'acquisizione di competenze specifiche per la lettura, l'analisi e l'interpretazione critica di un'opera letteraria. L'insegnante supporta lo studente nell'acquisizione degli strumenti necessari per entrare in contatto con il testo, individuare gli elementi di letterarietà e metterlo in relazione con la propria esperienza per poter esprimere un proprio parere, favorendo un percorso dinamico di crescita culturale e umana.

Da diversi anni nell'ambito degli studi sull'educazione e la didattica letteraria si è consapevoli del fatto che le generazioni più giovani, abituate alla frequente fruizione di immagini accompagnate da parole e musica, abbiano sviluppato maggiore familiarità con il linguaggio audiovisivo e che tale situazione abbia influenzato gli stili cognitivi e i processi formativi abituando a guardare e ad ascoltare più che a leggere, con ricadute spesso negative. Armellini osserva come alla crisi dell'insegnamento letterario abbia contribuito il forte impatto persuasivo del sistema delle comunicazioni di massa, che ha sottratto alla letteratura "sia le funzioni educative (trasmissione di modelli di comportamento, di valori etici, sociali, ecc.) sia le funzioni ludicoestetiche (invenzione di mondi possibili, espressione delle emozioni collettive, fascinazione del racconto)" $(2008,55)$. Da qui la necessità di avvicinarsi ai destinatari dell'insegnamento ampliando il corpus dei linguaggi letterari da proporre con testi basati sull'immagine, sulla musica e sulla contaminazione fra linguaggi, proprio per non escludere "i codici e i linguaggi di fondamentale importanza nella formazione del modo di sentire e di immaginare dei giovani, e non solo di essi" (2008, 59).

Solamente cercando connessioni con forme espressive apprezzate dai giovani sarà quindi possibile un'educazione letteraria idonea rispetto ai bisogni del discente, che non consisterà meramente nella trasmissione di nozioni ma contribuirà a costruire un sapere nuovo che verrà a crearsi dall'incontro tra $i$ testi proposti e l'immaginario dell'apprendente. Per stimolare tale incontro e mettere a frutto l'esperienza dello studente sarà opportuno ideare attività che possano mettere in connessione i temi di un testo letterario con "aree appropriate della conoscenza e della comprensione dello studente" per "'attivare' l'esperienza e creare i collegamenti necessari tra 'quello di cui parla il testo' e 'quello che questo elemento letterario significa per me"' altrimenti molti testi letterari che raccontano situazioni lontane 
dall'esperienza dello studente rimarranno inaccessibili (Zudič Antonič 621).

Oltre a ciò sarà fondamentale lavorare sull'aspetto dell'attualizzazione dei testi proposti e favorire un doppio scambio tra valori, concezioni e percezioni legate a epoche passate e rappresentazioni attuali, facendo emergere gli elementi di continuità: gli studenti acquisiranno gli strumenti per un approccio alle opere più tradizionali e spesso lontane dal loro immaginario, scoprendo in queste nuovi significati e fornendone nuove interpretazioni (Armellini2003, 30).

Affinché l'insegnamento letterario risulti efficace e apprezzato I'insegnante deve essere in grado, quindi, di far entrare la letteratura nella vita dello studente creando le condizioni per cui egli ne senta la necessità. Balboni, riguardo alla possibilità molto più immediata per uno studente di preferire la visione di un film tratto da un'opera letteraria rispetto alla lettura della stessa, rileva come sia indispensabile farlo riflettere sulle caratteristiche peculiari proprie dei due linguaggi - letterario e cinematografico - e sui meccanismi che hanno consentito la trasposizione da un linguaggio all'altro, per educarlo alla lettura sia del romanzo sia del film (2004,32-33). Un percorso didattico completo dovrebbe pertanto necessariamente orientarsi all'acquisizione di strumenti che permettano la comprensione di entrambe le tipologie di testi, sia sul piano contenutistico che formale, anche al fine di formare lettori e spettatori attivi che siano in grado di rapportarsi ai testi letterari tramite "processi di comparazione, contestualizzazione, interpretazione, che scaturiscono da una domanda di senso e portino a una costruzione cooperativa di significati" (Armellini 2003, 35).

Una delle possibilità per raggiungere questo obiettivo potrebbe configurarsi nella volontà dell'insegnante di proporre percorsi di comparazione tra adattamenti cinematografici e rispettive opere letterarie, orientati alla scoperta del funzionamento dei due linguaggi (configurazione della struttura narrativa, ruolo e funzioni dei personaggi, configurazione di spazio e tempo nel racconto scritto e in quello per immagini) per esplorare la posizione e il contributo del lettore e dello spettatore rispetto al messaggio trasmesso dal testo al fine di stimolare collegamenti, riflessioni e interpretazioni nuove e impreviste. Come evidenziato da Freddi, le trasposizioni da un'opera a un'altra con il passaggio da un codice linguistico a un altro, "lungi dal ridursi ad annacquamento della letteratura in altri prodotti semiotici, possono aiutare a scoprirne la specificità e la diffusività in diverse opere artistiche" (2003a, 71). L'individuazione di punti di convergenza o contrasto, di temi o di protagonisti che presentano tratti comuni, 
grazie all'immediatezza e al potere evocativo delle immagini renderà più accessibile l'avvicinamento alle opere letterarie tradizionali. II cinema narrativo, sia nelle sue forme più artistiche che in quelle più popolari e di genere, si presta a essere analizzato e sfruttato in tal senso perché conosciuto e amato, anche se negli ultimi decenni si trova a dover condividere questa posizione con le serie televisive.

Gli studenti dimostreranno interesse e risulteranno coinvolti quando saranno motivati e convinti di voler partecipare a un processo di educazione letteraria che risulterà in grado di soddisfare un loro bisogno e da cui trarranno piacere. Partendo dal modello della motivazione basato su dovere/piacere/bisogno e tenendo conto che un'educazione basata solamente sul dovere non risulta efficace, l'insegnante dovrebbe far scoprire agli studenti che la letteratura può soddisfare il loro bisogno di risposte a questioni che essi ritengono importanti e che sono legate ai quesiti fondamentali della vita, sui quali prima di loro hanno riflettuto i grandi intellettuali delineando i temi ricorrenti della letteratura. In più, riconoscere nei testi specificità strutturali e linguistiche, saperli collocare nel loro contesto ed essere in grado di valutare quali elementi rimangono attuali e quali caratteristiche attraggono la nostra attenzione, sono operazioni che suscitano una forma di grande piacere intellettuale (Balboni 2004, 15-18).

Un percorso formulato nel rispetto delle caratteristiche fino a qui discusse promuoverà l'utilizzo di strumenti e metodi che renderanno lo spettatore sempre più autonomo rispetto al messaggio veicolato, attivo nella ricezione e consapevole del prodotto artistico. Saranno così sviluppate e affinate competenze interscambiabili nello studio del cinema e della letteratura, visti i tratti di letterarietà e gli elementi sul piano della narrazione che opere letterarie e cinematografiche condividono. Freddi evidenzia inoltre come per il "giovane multimediale" la fruizione di film basati su opere letterarie possa risultare vantaggiosa in quanto

"si appassiona a una letteratura che sa reincarnarsi nel nostro tempo e giunge alla scoperta dei meccanismi dei linguaggi, anche non verbali e di quelli integrati e misti. Grazie a esperienze di tale natura il nostro giovane scopre quale linfa vitale sia la letteratura per comprendere l'immaginario elaborato nel tempo dai gruppi umani e quale nutrimento sia per nuove forme dell'immaginario, per la fantasia e la creatività. Il giovane scopre altresì, che con i suoi generi e le specifiche forme di scrittura, la letteratura offre una chiave per leggere e descrivere l'uomo e la vita. Scopre infine che la letteratura - frutto di un trattamento speciale della lingua - è uno di quei numerosi linguaggi della sfera estetica (arte, musica, danza, cinema, televisione ...) con cui 
si esprime la fantasia e la creatività dell'uomo del terzo millennio" (Freddi2003b, 5-6).

II rapporto tra letteratura e audiovisivo non dovrebbe quindi essere visto negativamente ma piuttosto come un'alleanza che offre la possibilità di studiare parallelamente i tratti più avvincenti di entrambi. Il punto di incontro più evidente tra cinema e letteratura è la narrazione. A mantenere alta l'attenzione del lettore/spettatore sono particolari episodi e punti di svolta organizzati nel discorso narrativo, che è possibile studiare e interpretare scoprendo ed esplicitando i procedimenti che la conducono. Per scoprirli e comprenderli si potrà guidare lo studente nell'analisi di un film, della sua struttura narrativa, confrontandolo con la versione scritta, rinviando alle specificità dei linguaggi letterario e filmico. Sono elementi condivisi dai due linguaggi la configurazione di fabula e intreccio, il ruolo del narratore, il punto di vista, il ritmo narrativo, i personaggi, le categorie di spazio e tempo, tutti da sfruttare in percorsi di studio volti ad approfondire la conoscenza di tali categorie nel racconto per immagini e in quello scritto. Saremo così in grado di offrire agli apprendenti gli strumenti per un approccio consapevole ad entrambe le forme artistiche, dando risposta ai loro bisogni e facendo loro scoprire il piacere della lettura e della comprensione dei meccanismi sottesi al funzionamento della narrazione cinematografica e letteraria.

\section{Perché e come utilizzare il cinema}

La visione di un film è un'esperienza emotiva e intellettuale appassionante, che suscita nello spettatore particolari meccanismi di identificazione con quanto viene mostrato sullo schermo. Per il coinvolgimento, l'intensità nella partecipazione come per i tratti di letterarietà e narratività, l'utilizzo del cinema nel contesto della didattica letteraria può essere considerato particolarmente stimolante e può contribuire allo sviluppo della capacità di decodifica e analisi di diverse forme di espressione artistica.

L'introduzione nella lezione di letteratura di momenti dedicati alla lettura delle immagini, parallelamente e a integrazione dei contenuti letterari tradizionali, apre la strada a un percorso di educazione all'immagine volto a una comprensione più profonda e strutturata del discorso audiovisivo, nonché per una sua ricezione attiva. L'accostamento del testo audiovisivo al testo scritto prendendo in esame, ad esempio, l'adattamento cinematografico di un'opera letteraria, concorre al raggiungimento dell'obiettivo di rafforzare la competenza letteraria intesa come acquisizione di abilità di lettura, 
comprensione, interpretazione e contestualizzazione, al fine di esprimere un giudizio critico rispetto a svariate tipologie di testi. L'interesse e la motivazione degli studenti saranno superiori quando l'argomento affrontato risulterà più vicino al loro mondo e ai prodotti che preferiscono, il film aggiungerà a quanto espresso nel testo letterario un contesto visivo, che potrà facilitare la comprensione del messaggio, incoraggiando ragionamenti riguardanti entrambe le modalità espressive.

Operativamente, l'utilizzo di film o spezzoni di film dovrebbe avvenire non in sostituzione ma a integrazione della lettura, per introdurre e presentare visivamente il contesto storico-tematico e l'ambientazione della vicenda narrata dall'opera letteraria come anche per accompagnare la lettura di un racconto attraverso confronti e riflessioni sulle scelte del regista e sulle eventuali modifiche (aggiunte o tagli) rispetto alla narrazione originaria. Proprio la ricerca di analogie e differenze e la riflessione sui motivi di tali rimodulazioni del racconto sono operazioni che richiedono una visione attiva e al contempo motivano lo studente alla lettura del testo scritto. Inoltre, la visione del film proposto dovrà essere sempre presentata e guidata dall'insegnante, sfruttando le strategie didattiche utilizzate nell'insegnamento della letteratura, al fine di convogliare l'interesse degli studenti e incoraggiare l'approccio critico da applicare non solamente ai testi studiati in classe ma anche a quelli presenti nella loro quotidiana esperienza di spettatori e lettori.

Tuttavia, film e romanzo rimangono opere autonome, da studiare in quanto tali, nel rispetto delle loro differenze concernenti i materiali espressivi e le modalità di racconto. Come sottolinea Bernardi $(9,13)$, se i film vengono visti come subordinati o persino antagonisti rispetto alla letteratura non potranno che presentarsi discordanti rispetto alle opere originarie e privi di una propria originalità. Uno studio comparativo in cui vengano presi in esame come testi equivalentemente validi, che funzionano secondo regole e processi differenti, potrà invece mettere in luce le caratteristiche di entrambi permettendone la vera comprensione.

\subsection{La narrazione come punto di incontro tra cinema e letteratura}

Opere cinematografiche e testi letterari, come già specificato, condividono l'esperienza narrativa. Individuando il funzionamento dei meccanismi della narrazione che letteratura e cinema condividono è possibile quindi comprendere come il contenuto narrativo venga espresso e comunicato nel contesto letterario e filmico. Un'analisi 
comparata delle due forme espressive è pertanto possibile proprio attraverso un approccio basato su concetti e categorie che rientrano nell'ambito disciplinare della narratologia ${ }^{7}$.

Partendo dalla distinzione tra storia e discorso come definita da Chatman (15), per cui la storia riguarda il piano del contenuto mentre il discorso si riferisce al piano dell'espressione, è possibile constatare che letteratura e cinema hanno la capacità di trasmettere $i$ medesimi contenuti utilizzando mezzi espressivi diversi: la parola scritta in letteratura, immagini in movimento accompagnate da una colonna sonora composta da parole (dialoghi e voci), rumori e musiche nel cinema.

Nel contesto degli adattamenti cinematografici le relazioni di scambio tra letteratura e cinema sono particolarmente evidenti. Tali connessioni sono immediatamente riconoscibili sia sul piano dei contenuti che quello più profondo dell'organizzazione e dell'espressione degli stessi. Accostare la letteratura al cinema può rivelarsi particolarmente utile proprio per la ricchezza dei diversi aspetti che è possibile sfruttare in ambito didattico: studiare un adattamento permette di indagare gli aspetti narrativi del testo letterario e di quello audiovisivo e di mettere in evidenza come una storia può essere riproposta o reinventata in un linguaggio diverso tramite una serie di strategie (sottrazione, condensazione, addizione, espansione, variazione, spostamento) messe in atto nel passaggio da una forma espressiva all'altra (Cortellazzo, Tomasi 29).

L'efficacia didattica del confronto fra testi e codici alla ricerca di elementi comuni e divergenze considerando, da un lato, le limitazioni poste dal linguaggio e dall'altro le scelte espressive degli autori del film, si configura nello sviluppo di un dibattito interpretativo che stimola e permette la riflessione critica e la presa di parola da parte degli studenti. Come sottolineano Cortellazzo e Tomasi (18),

"l'inevitabile e fertile logica del confronto ci porta a una maggiore conoscenza dei due testi: I'uno può infatti servire a far

7 II rapporto tra narrazione cinematografica e letteraria è stato esplorato soprattutto in questa prospettiva, con l'intento di analizzare forme e strutture della narrazione sulla base di metodi strutturalisti. A partire dalle analisi sulle funzioni narrative delle fiabe russe di V. Propp, diversi linguisti, semiologi, teorici del cinema, tra i quali R. Barthes, C. Bremond, S. Chatman, U. Eco, J. M. Lotman, G. Genette, A. J. Greimas, T. Todorov, hanno contribuito con le proprie ricerche a individuare i meccanismi di funzionamento e gli elementi minimali della narratività di un testo. 
maggiore luce sull'altro, a invitarci a seguire inediti percorsi di lettura, a rimettere in discussione quel che credevamo assodato. In sostanza ritornare a un romanzo dopo averne studiato - o perlomeno conosciuto - un adattamento può cambiare l'immagine che avevamo di quell'opera. In sintesi lo studio dell'adattamento può aiutare a capire come funzionano sia il racconto in sé e per sé (la narratività), sia il testo letterario e quello audiovisivo (in quanto particolari forme di espressione narrativa), sia infine, quel singolo romanzo e quel singolo film (come casi concreti e specifici)".

Sul piano della storia, l'attività didattica può essere incentrata sullo studio dei personaggi, degli ambienti e degli eventi, per trattare il senso delle riduzioni o variazioni effettuate dall'adattamento. Sul piano del discorso, il confronto diventa più complesso e include le categorie di narratore, punto di vista, spazio e tempo. Oltre a ciò, è possibile esaminare con gli studenti, partendo sia dal testo letterario che dal testo filmico, i contesti storico-sociale, culturale (correnti e movimenti letterari, artistici, ecc.) e produttivo in cui l'opera viene realizzata e che ne determinano la diffusione, per cercarne motivazioni e specificità. In sintesi, la scelta di operare un confronto permette di potenziare la conoscenza delle due tipologie di testo, mettere a fuoco le specificità che li caratterizzano acquisendo così una doppia competenza per la comprensione di entrambi.

\section{Come organizzare un percorso didattico}

Per la creazione di un percorso didattico di lettura e visione sarà fondamentale scegliere testi motivanti adatti alle conoscenze degli studenti e nella scelta considerare anche quelle tipologie più vicine alla loro esperienza, che però offrano spunto per analisi e riflessioni a più livelli, per un apprezzamento critico oltre a quello emotivo.

Il percorso didattico seguirà la struttura suddivisa nelle tre fasi di globalità, analisi e sintesi. L'accostamento globale al testo si concentrerà su attività (da svolgere autonomamente, in coppia, in gruppo) attraverso le quali lo studente sarà introdotto all'argomento affrontato e che lo aiuteranno a comprendere gradualmente il testo. $\grave{E}$ possibile partire da ipotesi iniziali basate sulle proprie conoscenze e da analogie con le proprie esperienze, in riferimento all'argomento del testo che si sta per affrontare o al suo contesto, per arrivare a concetti nuovi. In questa fase l'insegnante favorisce la motivazione e mantiene alta l'attenzione.

Nella fase di analisi lo studente osserva la nuova realtà, al fine di individuare punti di connessione e divergenza, decodificare e 
interpretare il messaggio dell'opera letteraria e filmica. Viene dedicata particolare attenzione all'aspetto formale del testo, di cui si evidenzieranno elementi caratteristici particolari da richiamare all'attenzione anche nello studio successivo di altri testi. L'insegnante supporterà gli studenti nell'individuazione dei diversi meccanismi di funzionamento dei due linguaggi e faciliterà lo sviluppo di abilità e competenze che possono servire nella lettura, nella comprensione e nell'interpretazione del testo, nonché nell'espressione - in forma orale e scritta - di giudizi critici.

L'obiettivo della fase di sintesi è quello di fissare le nuove conoscenze allargando il campo alla sintesi critica nel passaggio a una visione più ampia che include una riflessione sul contesto storicoletterario e sul genere, individuando, ad esempio, come lo stesso argomento sia stato affrontato in altri testi dello stesso periodo o dello stesso genere ed evidenziando l'appartenenza di elementi emersi durante l'analisi a macrocategorie generali. Questa fase comprenderà anche una valutazione personale dello studente, in cui esprime il proprio apprezzamento o meno per il testo studiato e riflette sull'utilità dell'analisi affrontata rispetto alle proprie esigenze, considerando se il testo abbia influenzato positivamente o modificato i suoi punti di vista e l'abbia stimolato a voler leggere o vedere altri testi. L'opera studiata potrà così entrare a far parte del bagaglio di conoscenze dello studente e arricchire la sua esperienza intellettuale. Rimangono comunque fondamentali l'apprezzamento e il godimento nella fruizione dei testi proposti in modo critico e a vari livelli, indice di una capacità di riflessione più matura.

\subsection{L'analisi del film}

Un pubblico che non possiede gli strumenti per leggere e interpretare le immagini di un prodotto audiovisivo, per capire come e perché sono state realizzate e a quale scopo sono state utilizzate soluzioni linguistiche, narrative o drammatiche particolari, è un pubblico facilmente suggestionabile dai modelli e dai valori proposti, siano questi positivi o negativi, reali o immaginari.

Una visione inconsapevole e passiva potrà sostituirsi a una fruizione attiva, solamente nel momento in cui lo spettatore sarà capace di analizzare tali testi in maniera distaccata, attraverso un approfondimento delle proprie conoscenze che aprirà lo spazio a un percorso di maturazione. In questa prospettiva si inserisce l'obiettivo principale dell'analisi di un testo audiovisivo: accrescere la consapevolezza critica e la capacità di decodificazione linguistica 
nonché il livello del gusto estetico, affinché lo studente, lettore e spettatore, sia capace di scelte libere e autonome.

L'obiettivo centrale dell'analisi sarà acquisire conoscenze più approfondite riguardo all'oggetto di analisi, cogliendone aspetti essenziali che allo spettatore/studente non è stato possibile rilevare alla prima visione perché catturato dalla narrazione e dall'opera nel suo complesso. L'operazione di analisi è vista come passaggio che permette la scomposizione (tramite attività di osservazione e descrizione) e ricomposizione del testo filmico (tramite attività di interpretazione e riflessione) evidenziandone i temi, la struttura, i meccanismi di funzionamento e i principi seguiti nella composizione. II film, come ogni opera artistica, rappresenta un'unità inscindibile di forma e contenuto e quindi lo studio dei contenuti comprenderà necessariamente anche l'analisi della forma in cui sono espressi, visto che è la combinazione e l'associazione di entrambi a generare significato.

Sulla base della suddivisione proposta da Rondolino e Tomasi (271-3), gli strumenti che si possono utilizzare nell'analisi di prodotti audiovisivi appartengono a tre categorie: strumenti descrittivi, tra i quali si collocano la sceneggiatura desunta, la segmentazione in sequenze ed episodi, le descrizioni dell'inquadratura; strumenti citazionali ovvero elementi estratti direttamente dal film quali sequenze, scene o inquadrature rilevanti per un commento; e strumenti documentari ovvero dati relativi al film ma esterni a esso, che saranno di supporto all'analisi e che riguardano la produzione, la realizzazione, commenti in interviste o altre informazioni che permettono di collocare il film nel contesto storico-artistico in cui è stato realizzato.

\subsection{Letteratura e cinema: un percorso parallelo}

Come già indicato, operazioni di collegamento e confronto saranno più agevoli nel caso degli adattamenti cinematografici di opere letterarie, ma vi è anche la possibilità di utilizzare film con sceneggiatura originale, rilevanti quando offrono una reinterpretazione degli stessi temi rispetto all'opera letteraria studiata o perché caratterizzati da aspetti stilistici specifici. II punto di partenza potrà essere tanto la lettura del racconto, quanto la visione del film.

Nel corso del confronto sarà possibile analizzare la trama, individuando elementi presenti e assenti nel testo letterario e nel testo filmico, in particolare in riferimento agli episodi narrativi in cui si articola il racconto, ai personaggi, all'ambientazione (spazio e tempo). Un'attività di questo genere permetterà di sintetizzare la struttura 
complessiva delle due opere attraverso la divisione in macro unità e di esaminare il ritmo narrativo, individuando punti di svolta, effetti sorpresa o altri espedienti che determinano l'andamento della trama e il procedere degli eventi. L'attività di comparazione dei personaggi è mirata all'individuazione delle soluzioni adottate nella rappresentazione del personaggio, in particolare nella visualizzazione degli stati d'animo, dei sentimenti e dei pensieri, che nel romanzo vengono espressi tramite descrizioni. L'analisi dell'ambientazione permette l'individuazione delle caratteristiche e delle funzioni dell'ambiente nella vicenda narrata, il rapporto che con l'ambiente instaurano i personaggi, il modo in cui questo viene rappresentato e il significato che assume.

L'obiettivo è individuare le scelte operate dal regista al fine di analizzare il nuovo contesto filmico e dare una motivazione a tali operazioni, soprattutto nel caso queste abbiano determinato variazioni rispetto ai temi o al messaggio centrale dell'opera originaria.

Procedendo, sarà possibile effettuare un'analisi comparativa sulla base delle categorie narratologiche che permetterà di individuare il narratore e l'istanza narrante, il punto di vista attraverso il quale le vicende sono narrate, al fine di scoprire eventuali soluzioni particolari adottate per scelta o necessità nel momento della trasposizione.

Successivamente sarà possibile procedere all'analisi del testo filmico sul piano delle immagini, delle loro caratteristiche, dettate da necessità tecniche e/o dovute alle scelte estetiche del regista, individuando il soggetto delle immagini, il tipo di rappresentazione (inquadratura), l'uso di illuminazione e colori, l'uso del montaggio, il funzionamento della colonna sonora (dialoghi, musiche, rumori).

In conclusione, l'insegnante guiderà lo studente nella riflessione sul rapporto personale con l'opera oggetto di analisi, su che cosa abbia significato per lui come spettatore, quali emozioni, riflessioni, idee, associazioni abbia suscitato e come siano stati ottenuti tali effetti.

Per stabilire un rapporto critico con il testo filmico è necessario ricordare sempre che il cinema utilizza l'immagine della realtà per costruirne una rappresentazione. In più è importante considerare che nel nostro caso lo spettatore è un soggetto apprendente che deve essere guidato dall'insegnante in un percorso di visione orientata allo svolgimento di attività di analisi e valutazione critica. Vanoye e GoliotLété (19-20) distinguono uno spettatore normale che assiste passivamente alla proiezione di un film, avendo come unico scopo lo svago e il piacere della visione, da uno spettatore attivo, analista, che assiste alla visione con finalità di studio. Per quest'ultimo la visione del 
film rientra nella sfera della riflessione e della produzione intellettuale, determinando un processo di distanziazione dalle immagini piuttosto che di identificazione. Tuttavia, siamo dell'opinione che l'analisi di un film in contesto didattico vada condotta attraverso un approccio che includa sia la partecipazione emotiva che il distacco critico. La prima permetterà di percepire intuitivamente ed emotivamente il messaggio, al contempo però, l'attenzione e l'osservazione saranno mantenute attive nel contesto dello svolgimento di attività che aiuteranno lo studente a riflettere su quanto visto.

Un percorso di analisi non dovrebbe togliere il piacere della visione di un film, dovrebbe invece favorire il passaggio a un livello più approfondito di conoscenza e comprensione dell'opera guidando lo studente verso una forma di piacere intellettuale più alto, che deriva proprio dall'individuazione dei meccanismi di funzionamento del testo. Sarà compito del docente guidare gli studenti nella riflessione finalizzata all'elaborazione e all'interpretazione delle immagini, attraverso il dibattito e il confronto delle diverse idee e opinioni e lo svolgimento delle attività.

\section{Percorsi didattici di letteratura italiana e cinema}

I percorsi didattici di seguito illustrati sono stati sviluppati per studenti universitari del corso di laurea in italianistica che studiano l'italiano come L2 o LS e che possiedono una competenza linguistica almeno di livello B1-B2. Attraverso la visione e l'analisi di testi (adattamenti di alcune opere letterarie per il cinema ma anche film con sceneggiature originali) e con l'apporto di ulteriore materiale autentico, lo studente è guidato in un percorso di studio in cui vengono affrontati argomenti che permettono di acquisire conoscenze relative alle componenti principali del linguaggio letterario e cinematografico, alle loro specificità, alle corrispondenze e differenze, alle opere e agli autori, al contesto storico-artistico, nonché di applicare e verificare tali conoscenze attraverso attività volte allo studio del percorso creativo alla base della realizzazione delle tipologie di testi proposte.

Al termine del percorso lo studente è in grado di dimostrare, attraverso attività di produzione orale e scritta, di aver acquisito gli strumenti per effettuare autonomamente un'analisi comparativa di testi filmici e opere letterarie e di saperne interpretare il messaggio ed esprimere adeguatamente un proprio giudizio.

Le attività sono incentrate su passi tratti da opere letterarie e filmiche, e mettono in evidenza alcune caratteristiche principali sul piano del contenuto e sul piano formale. Successivamente alla lettura di testi e alla visione di spezzoni di film sono previsti esercizi di 
comprensione, di analisi e di interpretazione. Agli studenti vengono inoltre fornite informazioni riguardanti l'autore e gli argomenti trattati, le specificità relative all'uso del linguaggio e il contesto storicoartistico, quando necessarie per lo svolgimento delle attività.

Per la predisposizione degli esercizi è stata utilizzata una pluralità di fonti come testi specialistici, antologie per lo studio della letteratura italiana, recensioni e commenti pubblicati in riviste e siti internet, utili a fornire informazioni relative agli argomenti trattati. Le attività puntano essenzialmente sulla comprensione testuale, l'espressione orale (riflessione e scambio di punti di vista) e l'espressione scritta, mentre è stata volutamente esclusa ogni tipo di attività esplicitamente grammaticale. II lavoro a coppie o in piccoli gruppi contribuisce solitamente a creare una buona atmosfera di cooperazione in classe e stimola la discussione e la consapevolezza tra gli studenti. Gli studenti sono incoraggiati a scoprire da soli gli aspetti più rilevanti mentre assistono alla visione di un film o leggono un testo. Tuttavia, ci sono momenti in cui si prevede che vengano date loro indicazioni esplicite e il docente può sempre guidare le risposte in caso di necessità. È prevista anche una parte di lavoro finalizzato allo sviluppo di un'autonomia nella ricerca delle nuove informazioni per dare allo studente la consapevolezza del suo apprendimento.

Il primo percorso didattico prevede una riflessione sull'esperienza dell'andare al cinema, in cui lo studente può mettere a confronto le proprie abitudini di spettatore con quelle descritte da Stefano Benni in uno dei racconti della raccolta /l bar sotto il mare nonché quelle rappresentate nel film Nuovo cinema Paradiso (1989) di Giuseppe Tornatore. Viene proposta la lettura del racconto II porno sabato dello Splendor, seguita dalla visione di una scena della durata di circa 5 minuti tratta dal film di Tornatore, in cui viene presentato il pubblico che frequenta la sala cinematografica del Cinema Paradiso e assiste alla proiezione di diversi classici del cinema. Attraverso lo svolgimento delle attività lo studente fa, inizialmente, delle previsioni rispetto ai contenuti del testo scritto osservando la copertina del libro ${ }^{8}$, successivamente legge il testo e ne verifica la comprensione analizzando ambienti e comportamenti dei personaggi, e riflettendo sull'utilizzo degli effetti comici da parte dello scrittore. In un secondo momento procede alla visione dello spezzone del film che poi confronta con il testo scritto individuando eventuali aspetti comuni dal

${ }^{8}$ Si tratta della copertina della prima edizione Feltrinelli (1987) che ritrae i personaggi avventori del bar e narratori dei diversi racconti, tra i quali è possibile riconoscere volti noti. 
punto di vista contenutistico (ambienti e personaggi). Infine, lo studente riflette sull'idea di cinema che emerge dal racconto e dal film paragonandola alla propria esperienza che descrive in un breve componimento scritto ${ }^{9}$.

II secondo percorso didattico è incentrato sul rapporto tra cinema e letteratura, in particolare sulle pratiche di adattamento e trasformazione di un testo scritto in un testo audiovisivo. L'attività è orientata all'ampliamento delle conoscenze degli studenti sulla strategia di riduzione come procedura di adattamento ma anche verso una riflessione più generale riguardo alle esigenze della narrazione per immagini, che solitamente determina la scelta di registi e sceneggiatori di optare per soluzioni narrative diverse rispetto alla letteratura. Inizialmente viene proposta la lettura di un brano tratto dal primo capitolo del romanzo di Umberto Eco II nome della rosa, più precisamente il passo nel quale vengono descritti gli avvenimenti del primo giorno, introdotto dal sottotitolo Dove Adso ammira il portale della chiesa e Guglielmo ritrova Ubertino da Casale, che ha una lunghezza di circa 5 pagine. Gli studenti svolgono alcune attività volte alla comprensione del testo che li guidano alla scoperta della tipologia e della strutturazione delle sequenze narrative contenute nel passo letto nonché del concetto di punto di vista. Successivamente viene proposta la visione della corrispondente sequenza del film II nome della rosa (1986), girato dal regista Jean-Jacques Annaud. Attraverso attività di analisi delle immagini viene chiesto agli studenti di riflettere su alcuni elementi relativi alle operazioni di adattamento del romanzo per il cinema (la riduzione) e sul passaggio dai meccanismi narrativi letterari (la descrizione) verso tecniche proprie del racconto per immagine. Viene poi incoraggiata una riflessione sull'effetto sorpresa nel cinema considerando come le informazioni tra istanza narrante, personaggi e spettatore vengono regolate al fine di creare effetti diversi (suspense, sorpresa). In conclusione, gli studenti propongono in

\footnotetext{
${ }^{9}$ Per la redazione delle attività è stata utilizzata l'unità didattica realizzata da Nives Zudič Antonič e presentata nel contributo dal titolo "Due realtà culturali, un unico modo di affrontare il testo letterario nella classe d'italiano", pubblicato nella rivista Inter E.O.I. numero 7 (2003), alle pagine 43-48. II materiale è stato rielaborato e integrato con alcune attività specifiche, ponendo l'attenzione soprattutto al confronto tra quanto rappresentato dalle immagini e quanto descritto nel racconto.
} 
un breve testo scritto una propria versione di adattamento del passo letto ${ }^{10}$.

Il terzo percorso didattico prevede invece un'analisi comparativa della rappresentazione e delle caratteristiche dei personaggi nei testi narrativi prendendo in esame un passo tratto dal romanzo /l Gattopardo di Giuseppe Tomasi di Lampedusa e le scene corrispondenti tratte dall'omonimo film realizzato da Luchino Visconti nel 1963. L'attività è orientata allo studio delle soluzioni che il racconto per immagini adotta per la necessità di mostrare la personalità dei protagonisti e rivelare pensieri ed emozioni senza la possibilità di ricorrere alla descrizione. In particolare, si propone agli studenti la lettura del passo che racconta l'entrata in società di Angelica, principale protagonista femminile, e la visione del relativo spezzone del film. Le attività sono volte alla comprensione dei due testi nonché all'esame delle modalità con cui il personaggio viene presentato nel testo scritto e nel film, considerando gli effetti prodotti rispettivamente sul lettore e sullo spettatore. Dopo la lettura viene chiesto agli studenti di scrivere un breve profilo della protagonista femminile tenendo conto di quanto emerge dal romanzo per confrontarlo in un secondo momento con quanto mostrato dalle immagini. Particolare attenzione viene dedicata alla descrizione e alla visualizzazione degli stati d'animo e delle reazioni dei personaggi, nonché alle sensazioni che attraverso le immagini vengono trasmesse allo spettatore ${ }^{11}$.

\footnotetext{
${ }^{10}$ Per la redazione delle attività sono stati utilizzati i testi e alcuni esercizi contenuti nell'antologia italiana per il biennio di M. E. Battaglia, A. Arato e D. Corno dal titolo Fare testo (Paravia, 1992, 213-220) nonché nell'antologia per lo studio della letteratura italiana di C. Siviero e A. Spada, Nautilus. Alla scoperta della letteratura italiana. Dalla fine dell'Ottocento alla fine del Novecento, (Zanichelli, 2011, 762-765), ai quali sono state aggiunte attività originali di analisi del testo cinematografico. È stato inoltre incluso un passo tratto dall'intervista di F. Truffaut ad A. Hitchcock contenuta nel volume II cinema secondo Hitchcock di F. Truffaut (Pratiche, 1985, 58-59).

${ }^{11}$ Per la redazione della parte introduttiva alle attività si è fatto riferimento alla scheda sul personaggio contenuta nella già citata antologia italiana per il biennio Fare testo (pagine 932-941). Il passo del romanzo di Tomasi di Lampedusa e alcuni esercizi di comprensione del testo sono stati tratti dall'antologia Nautilus. Alla scoperta della letteratura italiana. Dalla fine dell'Ottocento alla fine del Novecento (pagine 703-706) e sono stati integrati con attività originali relative all'analisi del testo cinematografico.
} 


\section{Considerazioni conclusive}

Le attività didattiche descritte sono state ideate in linea con un'idea di ampliamento e rinnovamento del corpus di linguaggi della letteratura, oggetto di studio nell'ambito dei curricoli tradizionali di educazione alla letteratura. L'approccio congiunto, che riconosce alla letteratura e al cinema specificità e qualità proprie, permette di conoscere meglio e trarre vantaggio da entrambi. Partendo dalla necessità di comprendere i componenti del linguaggio letterario e del linguaggio cinematografico, nonché le caratteristiche del testo narrativo e i meccanismi di funzionamento, le attività ideate favoriscono la creazione di rimandi e connessioni tra le due forme espressive e ne favoriscono l'apprendimento.

La nostra esperienza dell'utilizzo in classe dei materiali illustrati si è rilevata particolarmente positiva: oltre a suscitare partecipazione e collaborazione da parte degli studenti nel corso delle discussioni, ha potenziato la motivazione e ha favorito un atteggiamento esplorativo nei confronti dei testi attivando l'esperienza personale degli studenti e portandoli a porsi nuove domande.

II percorso ha quindi favorito il raggiungimento della meta generale dell'educazione rappresentato dall'insegnamento/ apprendimento a leggere un testo letterario nel rispetto di tre passaggi fondamentali descritti da Balboni (2003, 143; 2004, 33), Armellini (1987, 95-96) e Colombo (9-10):

a. individuare e comprendere il significato intrinseco del testo a diversi livelli riflettendo sul contesto passato in cui è stato prodotto e sulla valenza che assume per il lettore nel contesto attuale, mantenendo collegamenti con linguaggi più familiari e allargando il campo delle tipologie di testi fruiti al fine di ottenere curiosità, coinvolgimento e invogliare a leggere;

b. individuare le caratteristiche estetico-formali per cui un testo è considerato letterario, le regole e le convenzioni seguite dall'autore, partendo da quello che lo studente già conosce e sa mettere in pratica;

c. interpretare ed esprimere, argomentando, un giudizio critico confrontandolo con altri giudizi, altri testi e un più vasto contesto letterario, per far emergere riflessioni personali e infine far diventare il testo patrimonio del lettore/spettatore.

Si configura in questa prospettiva la possibilità di mettere in atto un'educazione alla lettura - nel suo più ampio significato - di opere letterarie e cinematografiche, nonché allo sviluppo della capacità critica, affiancando lo studente, lettore e spettatore, in un percorso di fruizione consapevole e di migliore comprensione. La lettura di testi 
letterari assieme alla visione di film, indirizzata all'individuazione di tratti caratteristici e finalizzata alla rielaborazione cognitiva mediante attività di analisi, riformulazione e interpretazione, apre lo spazio a nuove opportunità, rafforzando o cambiando punti di vista e contribuendo al consolidamento della materia oggetto di apprendimento.

\section{Bibliografia:}

Armellini, Guido. Come e perché insegnare letteratura. Strategie e tattiche per la scuola secondaria. Bologna: Zanichelli, 1987.

-"Letteratura e altro. Tra aperture teoriche, trappole burocratico-pedagogiche e artigianato didattico." Scuola e Lingue Moderne 41.7-8 (2003): 26-36.

----------La letteratura in classe. L'educazione letteraria e il mestiere dell'insegnante. Milano: Unicopli, 2008.

Balboni, Paolo E. Le sfide di babele. Insegnare le lingue nelle società complesse. Torino: UTET, 2003.

"Non scholae sed vitae. Educazione letteraria e didattica della letteratura." Educazione letteraria e nuove tecnologie. A cura di Paolo E. Balboni. Torino: UTET, 2004: 5-56.

Bernardi, Sandro. Fare scuola con i film. Guida ragionata a $130 \mathrm{film}$ in videocassetta divisi per materia. Firenze: Sansoni, 1991. Chatman, Seymour. Storia e discorso. La struttura narrativa nel romanzo e nel film. Traduzione di Elisabetta Graziosi. Milano: Net, 2003.

Colombo, Adriano. "A che punto è l'insegnamento di letteratura." La letteratura per unità didattiche. Proposte e metodi per l'educazione letteraria. A cura di Adriano Colombo. Firenze: La Nuova Italia, 2000.

Cortellazzo, Sara e Dario Tomasi. Letteratura e cinema. Bari: Laterza, 2008.

Freddi, Giovanni. La letteratura. Natura e insegnamento. Milano: Ghisetti \& Corvi, 2003a.

"Letteratura e letterature per il giovane del terzo millennio."

Scuola e Lingue Moderne 41.7-8 (2003b): 3-13. Rondolino, Gianni e Dario Tomasi. Manuale del film. Linguaggio, racconto, analisi. Torino: UTET, 1995.

Vanoye, Francis e Anne Goliot-Lété. Introduzione all'analisi del film. Traduzione di Dario Buzzolan. 2a ed. Torino: Lindau, 2003. 
Zudič Antonič, Nives. "Educazione letteraria per lo sviluppo della consapevolezza culturale". Annales. Anali za istrske in mediteranske študije. Annali di Studi istriani e mediterranei. Annals for Istrian and Mediterranean Studies 27.3 (2017): 611628.

\section{Književnost i film: upotreba filma u metodici nastave italijanske književnosti}

Film je jedna od najsugestivnijih narativnih formi, naročito za studente koji odrastaju u kontaktu sa pretežno vizuelnom kulturom. Korišćenje iskustava i sposobnosti studenata $u$ vezi sa dekodifikovanjem audiovizuelnih poruka u motivacione svrhe odlučujući je korak u pripremi i primeni nastavnih programa nastave književnosti.

Cilj ovog članka je da ilustruje značaj, motive i korist upotrebe filma u nastavi književnosti, nudeći alternativne programe i metode u odnosu na tradicionalnu metodiku nastave. Osim toga, biće navedene prednosti paralelnog proučavanja književnih tekstova i filmova vezanih za italijanski kulturni milje koji se mogu iskoristiti da se analiziraju i prodiskutuju predstave i slike, ohrabrujući pristupanje različitim vrstama tekstova i načinima izražavanja, koji su istovremeno i predmet proučavanja i umetnička dela u kojima se može uživati.

Nastavni program se zasniva na uporednom proučavanju formalnih i estetskih aspekata književnih dela i filmova s ciljem da se uoče zajednički elementi i interakcija između njih. Na taj način se pasivno gledanje filmova zamenjuje aktivnim pristupom $i$ to je ono što predstavlja osnovnu prednost paralelnog proučavanja književnosti i filma: povećava se kritička svest i sposobnost lingvističke dekodifikacije, kao i estetska percepcija studenata. Krajnji cilj je da se čitaoci i gledaoci edukuju kako bi bili u stanju da donose autonomne odluke u obilju audio-vizuelnih poruka koje su odlika savremenog društva.

Ključne reči: italijanska književnost, italijanski film, filmske adaptacije, nastava književnosti, metodika nastave 